\title{
Haemochromatosis: Facilitating Blood Donation as an Alternative to Management with Venesection for Patients in Morecambe Bay
}

\author{
Jennifer Sullivan, MA, BSc (Hons), RN, Clinical Lead, Day Treatment Units RLI \& WGH
}

\section{BACKGROUND}

Genetic Haemochromatosis (GH) is one of the most frequent genetic disorders found in people of Northern European descent; it is characterised by a continued absorption of iron from the intestine in excess of that required for normal erythropoiesis. ${ }^{1}$ As the body has no mechanism for removing iron, this excess iron can accrue in the liver, pancreas, heart, endocrine glands, and joints. In advanced disease iron build up in the body tissues may cause pervasive tissue damage including cirrhosis and diabetes mellitus ${ }^{2}$. Updated guidelines by the British Committee for Standards in Haematology state that at diagnosis all fit $\mathrm{GH}$ patients should undergo weekly venesection until Serum Ferritin (SF) is $20-30 \mu \mathrm{g} / 1$ and transferrin saturation (Tsat) $<50 \% .^{2}$ Once excess iron has been removed, iron will begin to re-accumulate unless venesection recurs periodically. During this maintenance phase, patients are to be venesected as required, preferably at a blood donation centre, to maintain normal full blood count (FBC), Serum Ferritin (SF) $<50 \mu \mathrm{g} / \mathrm{l}$ and transferrin saturation (Tsat) $<50 \% .^{2}$

There is no inherent contraindication why patients with GH cannot be considered as blood donors. NHS Blood and Transplant (NHSBT) have accepted blood from GH patients since 2001 and have a patient information leaflet outlining how $\mathrm{GH}$ patients can register as blood donors. ${ }^{3}$ Examples exist that have been shown to be successful in enabling GH patients to transition to blood donation. Marrow et al. described the introduction of a dedicated clinic in the UK in 2011 to facilitate the transition to blood donation for $\mathrm{GH}$ patients. ${ }^{4}$ Mishra et al describe a patient self-management model in New Zealand whereby GH patients who become blood donors are discharged to the care of their General Practitioner, ${ }^{5}$ however, a recent survey led by the University of Surrey on behalf of Haemochromatosis UK found that $90 \%$ of $\mathrm{GH}$ patients in the UK are managed with venesection. ${ }^{6}$

Patients with GH regularly attend the medical Day Treatment Units at our hospitals (Day Treatment Unit, Royal Lancaster Infirmary (RLI), Dunmail Treatment Centre Westmorland General Hospital (WGH) and Oncology, Furness General Hospital (FGH)) for venesection to manage their condition. Any blood removed is discarded; meanwhile, other patients are attending the same departments to receive blood and platelet transfusions. The contrast between disposing of blood while simultaneously facing patients who need this valuable resource is stark. The staff in our venesection departments arguably have the most frequent contact with GH patients, not only when they attend for venesection and/or blood tests, but also when reviewing the results of those blood tests and informing the patient of results. They thus have ongoing contact with the patient that occurs between the medical review appointments with the patient's clinical team, which may only occur annually. They are ideally placed to assist GH patients to transition to blood donation; hitherto, they have had no means of doing so. University Hospitals of Morecambe Bay NHS Foundation Trust (UHMBT) has developed a new Standard Operating Procedure (SOP) that will enable venesection staff to facilitate blood donation as an alternative choice for patients with $\mathrm{GH} .{ }^{7}$ This article does not address the ongoing medical management of $\mathrm{GH}$ patients, rather, it outlines key features that underpin the development of the SOP, and the role (and limitations of the role) of venesection staff within the process of the patient transition from venesection to blood donation as an alternative way to manage their GH.

\section{KEY FEATURES}

The following were highlighted during the development of the new SOP:

1. Identifying which patients are eligible. Patients need to be in the maintenance phase of their condition before NHSBT will consider accepting them as a blood donor. The responsibility for deciding if a patient is suitable to manage their $\mathrm{GH}$ with blood donation remains with the medical team. The SOP outlines the criteria that enables venesection staff to identify if a $\mathrm{GH}$ patient is in maintenance phase prior to contacting the patient's clinical team for medical confirmation that blood donation is a viable option for the patient.

2. Identifying which patients not eligible. NHSBT require that $\mathrm{GH}$ patients are free from organ damage due to iron overload and not be taking medication for their GH (oral iron chelators). ${ }^{3}$ In addition, the removal of blood for some patients is physically challenging and this necessitates that they are managed in a hospital environment. For example, they require their venesections to be conducted isovolemically or they have difficult venous access. Importantly the SOP makes clear for our venesection staff which GH patients are not eligible, as well as those that are.

3. Patient role in managing their $\mathbf{G H}$. Venesection staff have historically informed patients when venesection is needed after reviewing blood results. This has historically placed patients in a dependent role, having their condition managed for them, it has also contributed to a perception that this style of relationship between patients and venesection staff is necessary for all patients. Choosing blood donation as a way of managing $\mathrm{GH}$ enables the patient to have a more active role in managing their condition.

4. Coexisting conditions or medication. GH patients must meet the same screening criteria as any other blood donor. The responsibility of who can be accepted as a blood donor remains with NHSBT. Many people (patients and staff) assume they are 
not eligible to be blood donors because they take medication or have coexisting conditions; they may even have been informed of this many years ago by NHSBT. NHSBT is a separate NHS Organisation in the UK; it has its own organisational structures and staff separate from UHMBT or any other NHS Trust. A history of an absence of day to day networking between frontline NHSBT and UHMBT staff has contributed to a lack of accurate knowledge of current screening criteria for blood donation. The SOP outlines a means for venesection staff to answer initial queries from patients regarding NHSBT current screening criteria for blood donation.

5. Ongoing review of the patient's condition. NHSBT may accept GH patients as blood donors but does not offer ongoing review or management of their GH. Importantly the SOP ensures that GH patients are not 'lost' from medical review if they become blood donors.

6. Patient preference. Blood donation in the UK is entirely voluntary. In addition, patients may develop positive relationships with venesection department staff and wish this to continue. The SOP is not intended to compel those patients to become blood donors who wish to continue with venesections; it is intended that for those patients who wish to change from venesection and become a blood donor, UHMBT venesection staff are able to facilitate the process and ensure it is safe.

\section{PATIENT INFORMATION}

The process of developing the SOP highlighted a need for a patient information leaflet. UHMBT patient information standards state that patients are less likely to need to contact our departments with questions if they are given appropriate written information. ${ }^{8}$ The patient information leaflet ${ }^{9}$ is intended to give the patient written information that supports any discussion with staff; it is not a replacement for such discussion. It contains a patient specific section for staff to complete before it is given to the patient to take away. The patient then has personalised written advice on how often they need to donate blood to manage their condition, and how often they should have blood tests. Importantly, it includes guidance on how GH patients resume venesection if they can no longer continue as a blood donor.

\section{BENEFITS OF BLOOD DONATION VERSUS VENESECTION FOR GH PATIENTS}

The following benefits are envisioned as arising from UHMBT staff facilitating a change to blood donation as a means for patients to manage their condition:

1. An increase in patient choice. Many patients express a wish that their blood be used for someone who needs it. Venesection staff will now be able to explore if blood donation is a viable option for an individual patient and facilitate the transition from venesection. The patient gains more control over how they manage their condition, playing a more proactive role.
2. NHSBT gain more blood donors. More blood is made available for others who need transfusion.

3. An increase in staff expertise. The development of closer links with NHSBT and involvement of staff in facilitating patient transition from venesection will contribute to a greater understanding and awareness of blood donation as a management method for $\mathrm{GH}$.

4. Financial saving. A decrease in venesection appointments will free up staff time for other appointments and reduce costs.

\section{PILOT PERIOD AND AUDIT}

Changing from venesection to blood donation is a process. It involves discussion and contact with different members of the teams involved in the patient's care: venesection department staff, the patient's clinical team and NHSBT. More than one member of venesection staff may be involved in the process for an individual patient. It is important that this process is safe and facilitated in the best way for the patient. A tracking process, Haemochromatosis Blood Donor Log, has been incorporated into the SOP to ensure that the patient's transition from venesection to blood donation can be monitored and progressed appropriately. In addition, the SOP has also been given a short review date of 1 year, to ensure timely review. During this pilot period (beginning March 2019) audit is planned to occur at 3 months, 6 months and 12 months. The Haemochromatosis Blood Donor Log will also facilitate audit and review during the pilot period.

\section{MAKING THE CHANGE}

A training process has been devised for staff in our venesection departments to support the introduction of the SOP. While venesection staff do not hold ultimate responsibility for deciding who is eligible (this remains with the patient's clinical team) or who can be accepted as a blood donor (this remains with NHSBT), they have an important role in providing information to the patient, communicating with the patient's clinical team and ensuring the patient remains on the correct Access Plan to enable ongoing review of their GH. Relevant documents and resources to support staff are available on a dedicated webpage 'Haemochromatosis Blood Donors' on the Medicine section of UHMBT intranet.

\section{CONCLUSIONS}

It is difficult to predict how many of our GH patients will wish to change from venesection to blood donation to manage their condition. We will only begin to be able to estimate this at the end of the pilot period. The criteria in the SOP of which GH patients are eligible to manage their condition with blood donation has been consciously set conservatively in order to minimise any risk for $\mathrm{GH}$ patients during the pilot period. However, even a small shift in the percentage of $\mathrm{GH}$ patients who are managed by blood donation or venesection cited earlier ${ }^{6}$ has the potential to make a big difference, for individual $\mathrm{GH}$ patients and those who require blood products. UHMBT 
venesection teams are now in a position to begin contributing to such a shift.

Acknowledgement is gratefully offered to the following UHMBT staff:

- For their contribution to consultations on development of the SOP: Jill Livingston, Transfusion Practitioner; Anna-Marie Jesson, (formerly manager) of NHSBT Blood Donor Suite, RLI; Dr Mane, Consultant Haematologist; Dr Colin Brown, Consultant Gastroenterologist; Julie Bethell and Katy Kelton, Clinical Nurse Specialists Haematology; Jayne Sharkey, Advanced Nurse Practitioner Gastroenterology; Dr Oo, Consultant Haematologist; Dr Andrew Higham, Medical Director; Louise Pucknell, Patient Safety Manager; Jayne Bickerstaff, Ward Manager, Oncology FGH; Carolyn Hicks, Ward Manager Day Treatment Unit RLI and Dunmail Treatment Centre WGH; Angela Preston, RN Day Treatment Unit RLI.

- For peer review on the patient information leaflet: Jill Livingston, Transfusion Practitioner; AnnaMarie Jesson, (formerly manager) of NHSBT Blood Donor Suite, RLI; Dr Colin Brown, Consultant Gastroenterologist; Dr Oo, Consultant Haematologist; Dr Howarth, Consultant Haematologist; Jayne Bickerstaff, Ward Manager, Oncology FGH; Louise Pucknell, Patient Safety Manager; Melanie Woolfall, Associate Director of Nursing.

\section{REFERENCES}

1. Pietrangelo A, Hereditary haemochromatosis: pathogenesis, diagnosis and treatment, Gastroenterology 2010; 139: 393-408.

2. Fitzsimmons EJ, Cullis JO, Thomas DW, Tsochatzis E, Griffiths, WJH, on behalf of British Society for Haematology. Diagnosis and therapy of genetic haemochromatosis (review and 2017 update), British Journal of Haematology 2018;181:293-303.

3. Akhtar, N. NHS Blood and Transplant (2018) Information for Donors with Haemochromatosis. Available from https://nhsbtdbe.blob.core. windows.net/umbraco-assets-corp/14879/inf9352information-for-donors-with-haemochromatosisdl-v1.pdf (accessed on 11.3.19).

4. Marrow B, Clarkson J, Chapman CE. Masson S. Facilitation of blood donation amongst haemochromatosis patients. Transfusion Medicine 2015;25:239-242.

5. Mishra S, Sim D, and Flanagan P. Haemochromatosis: evaluating the effectiveness of a novel patient self-management approach to venesection as blood donation. New Zealand Medical Journal 2017;130(1457):26-33.

6. Smith,KJ, Fife-Schawa C,Dibba B, Griffiths W, on behalf of Haemochromatosis UK (2018). Living with the impact of iron overload: report from a large survey of people with haemochromatosis. Available from https://haemochromatosis.org.uk/patient_ report_summary/ (accessed 11.3.19).

7. University Hospitals of Morecambe Bay NHS Foundation Trust. Referring and monitoring of haemochromatosis patients who become blood donors. 2019.

8. University Hospitals of Morecambe Bay NHS Foundation Trust. Patient information leaflet handbook Version 1.6, updated January 2018.

9. University Hospitals of Morecambe Bay NHS Foundation Trust. Haemochromatosis: Managing your condition with blood donation, 2019. Available from: https://www.uhmb.nhs.uk/ files/7915/5197/6733/PIL115_PLAIN_TEXT_-_ Haemochromatosis_-_managing_your_condition_ with_blood_donation.pdf (accessed 11.3.19).

Correspondence to: jennifer.sullivan@mbht.nhs.uk

\section{GP and Consultant badminton match on 17/03/19 in Lancaster}

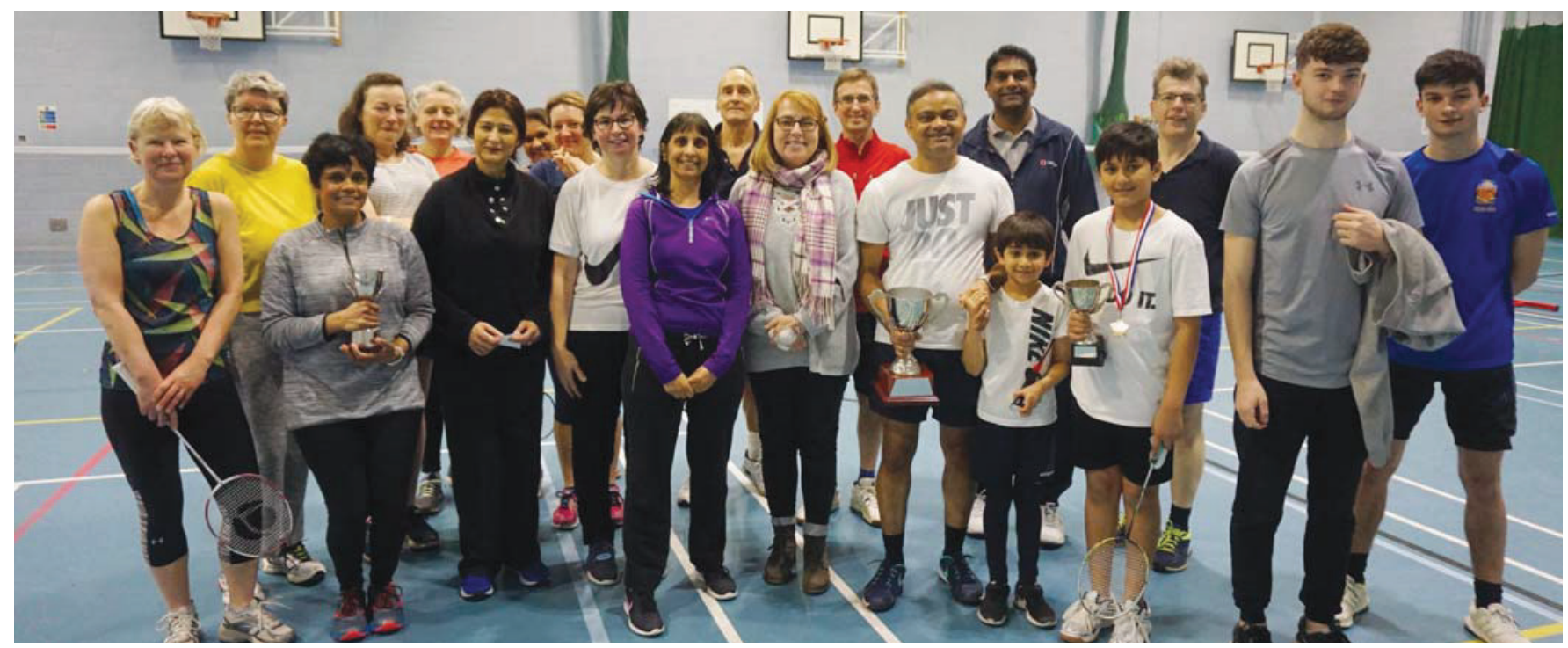

